\title{
On graphs double-critical with respect to the colouring number
}

\author{
Matthias Kriesell1|k \\ Anders Sune Pedersen $2,3 \|^{\prime}$ \\ 1 Department of Mathematics, Ilmenau University of Technology, Germany. \\ 2 Department of Mathematics and Computer Science, University of Southern Denmark, Odense, Denmark. \\ 3 Research Clinic on Gambling Disorders, Aarhus University Hospital, Denmark.
}

received $21^{\text {st }}$ Oct. 2012, revised $2^{\text {nd }}$ Jan. 2015, accepted $18^{\text {th }}$ Aug. 2015.

The colouring number $\operatorname{col}(G)$ of a graph $G$ is the smallest integer $k$ for which there is an ordering of the vertices of $G$ such that when removing the vertices of $G$ in the specified order no vertex of degree more than $k-1$ in the remaining graph is removed at any step. An edge $e$ of a graph $G$ is said to be double-col-critical if the colouring number of $G-V(e)$ is at most the colouring number of $G$ minus 2 . A connected graph $G$ is said to be double-col-critical if each edge of $G$ is double-col-critical. We characterise the double-col-critical graphs with colouring number at most 5 . In addition, we prove that every 4-col-critical non-complete graph has at most half of its edges being double-col-critical, and that the extremal graphs are precisely the odd wheels on at least six vertices. We observe that for any integer $k$ greater than 4 and any positive number $\epsilon$, there is a $k$-col-critical graph with the ratio of double-col-critical edges between $1-\epsilon$ and 1 .

Keywords: graph colouring, graph characterizations, degenerate graphs, colouring number, double-critical graphs

\section{Introduction}

All graphs considered in this paper are assumed to be simple and finite ${ }^{(i)}$ The cycle on $n$ vertices is denoted by $C_{n}$. The complete graph $K_{n}$ on $n$ vertices is referred to as an $n$-clique. Let $G$ denote a graph. The number of vertices in a largest clique contained in $G$ is denoted by $\omega(G)$. The vertex-connectivity of $G$ is denoted by $\kappa(G)$. The number of vertices and edges in $G$ is denoted by $n(G)$ and $m(G)$, respectively. Given a vertex $v$ in $G, N(v, G)$ denotes the set of vertices in $G$ adjacent to $v$; $\operatorname{deg}(v, G)$ denotes the cardinality of $N(v, G)$, and it is referred to as the degree of $v$ (in $G$ ). A vertex of degree 1 is referred to as a leaf. The minimum degree and maximum degree of $G$ is denoted $\delta(G)$ and $\Delta(G)$, respectively. Given a subset $S$ of the vertices of $G$, the subgraph of $G$ induced by the vertices of $S$ is denoted by $G[S]$, and we let $N(S, G)$ denote the set $\cup_{s \in S} N(s, G) \backslash S$. The square of a graph $G$, denoted by $G^{2}$, is the graph obtained from $G$ by adding edges between any pair of vertices of $G$ which are at distance

\footnotetext{
*Email: matthias.kriesell@tu-ilmenau.de

†Email: aspeimada.sdu.dk

(i) The reader is referred to [1] for definitions of graph-theoretic concepts used but not explicitly defined in this paper.

1365-8050 @ 2015 Discrete Mathematics and Theoretical Computer Science (DMTCS), Nancy, France
} 
2 in $G$. Given two graphs $H$ and $G$, the complete join of $G$ and $H$, denoted by $G+H$, is the graph obtained from two disjoint copies of $H$ and $G$ by joining each vertex of the copy of $G$ to each vertex of the copy of $H$. The chromatic number of $G$ is denoted by $\chi(G)$, while the list-chromatic number of $G$ is denoted by $\chi_{\ell}(G)$. Let $\psi$ denote some graph parameter. An edge $e$ of $G$ is said to be double- $\psi$-critical if $\psi(G-V(e)) \leq \psi(G)-2$. A connected graph $G$ is said to be double- $\psi$-critical if each edge of $G$ is double- $\psi$-critical. For brevity, we may also refer to double- $\chi$-critical edges and graphs as, simply, doublecritical edges and graphs, respectively.

The introduction of the concept of double- $\psi$-critical graphs in [12] was inspired by a special case of the Erdős-Lovász Tihany Conjecture [2], namely the special case which states that the complete graphs are the only double-critical graphs. We refer to this special case of the Erdôs-Lovász Tihany Conjecture as the Double-Critical Graph Conjecture. The Double-Critical Graph Conjecture is settled in the affirmative for the class of graphs with chromatic number at most 5 , but remains unsettled for the class of graphs with chromatic number $k$, for each value of $k \geq 6$. [4, 11, 16, 17]. Using the computer programs SAGE [15] and geng [10], we verified the Double-Critical Graph Conjecture for all graphs on at most 12 vertices (see [13]).

In [12], it was proved that if $G$ is a double- $\chi_{\ell}$-critical graph with $\chi_{\ell}(G) \leq 4$, then $G$ is complete. It is an open problem whether there is a non-complete double- $\chi_{\ell}$-critical graph with list-chromatic number at least 5 .

The double- $\kappa$-critical graphs, which in the literature are referred to as contraction-critical graphs (since the vertex-connectivity drops by one after contraction of any edge), are well-understood in the case where $\kappa$ is 4 . Some structural results have been obtained for contraction-critical graphs with vertex-connectivity 5. (See [6, Sec. 4] for references on contraction-critical graphs.)

Bjarne Tof ${ }^{(i i)}$ posed the problem of characterising the double-col-critical graphs. Here col denotes the colouring number which is defined in the paragraph below.

In this paper, we characterise the double-col-critical graphs with colouring number at most 5 .

In the remaining part of this section, we define the colouring number and present some fundamental properties of this graph parameter.

The colouring number of a graph. Suppose that we are given a non-empty graph $G$ and an ordering $v_{1}, \ldots, v_{n}$ of the vertices of $G$. Now we may colour the vertices of $G$ in the order $v_{1}, \ldots, v_{n}$ such that in the $i$ th step the vertex $v_{i}$ is assigned the smallest possible positive integer which is not assigned to any neighbour of $v_{i}$ among $v_{1}, \ldots, v_{i-1}$. This produces a colouring of $G$ using at most

$$
\max _{i \in\{1, \ldots, n\}} \operatorname{deg}\left(v_{i}, G\left[v_{1}, \ldots, v_{i}\right]\right)+1
$$

colours. Taking the minimum over the set $\mathrm{S}_{n}$ of all permutations of $\{1, \ldots, n\}$, we find that the chromatic number of $G$ is at most

$$
\min _{\pi \in \mathrm{S}_{n}}\left\{\max _{i \in\{1, \ldots, n\}} \operatorname{deg}\left(v_{\pi(i)}, G\left[v_{\pi(1)}, \ldots, v_{\pi(i)}\right]\right)\right\}+1
$$

The number in (1) is called the colouring number of $G$, and it is denoted by $\operatorname{col}(G)$. The colouring number of the empty graph $K_{0}$ is defined to be zero. By $1, \operatorname{col}(G) \leq \Delta(G)+1$ for any graph $G$.

(ii) Private communication to the second author from Bjarne Toft, Odense, August 2008. 
The colouring number was introduced by Erdős and Hajnal [3], but equivalent concepts were introduced independently by several other authors. It can be shown (see, for instance, [19]) that the colouring number of any non-empty graph $G$ is equal to

$$
\max \{\delta(H) \mid H \text { is an induced subgraph of } G\}+1
$$

and that the colouring number can be computed in polynomial time [9]. The non-empty graphs with colouring number at most $k+1$ are also said to be $k$-degenerate [8]. Thus, a non-empty graph $G$ is $k$-degenerate if and only if there is an ordering of the vertices of $G$ such that when removing the vertices of $G$ in the specified order no vertex of degree more than $k$ in the remaining graph is removed at any step.

The colouring number is monotone on subgraphs, that is, if $F$ is a subgraph of a graph $G \operatorname{then} \operatorname{col}(F) \leq$ $\operatorname{col}(G)$. For ease of reference, we state the following elementary facts concerning the colouring number of graphs.

Observation 1 For any graph $G$,

(i) $\operatorname{col}(G)=0$ if and only if $G$ is the empty graph,

(ii) $\operatorname{col}(G)=1$ if and only if $G$ contains at least one vertex but no edges,

(iii) $\operatorname{col}(G)=2$ if and only if $G$ is forest containing at least one edge, and

(iv) $\operatorname{col}(G) \geq 3$ if and only if $G$ contains at least one cycle.

A graph $G$ is said to be $k$-col-critical, or, simply, col-critical, if $\operatorname{col}(G)=k$ and $\operatorname{col}(F)<k$ for every proper subgraph $F$ of $G$. Similarly, a graph $G$ is said to be $k$-col-vertex-critical, or, simply, col-vertexcritical, if $\operatorname{col}(G)=k$ and $\operatorname{col}(F)<k$ for every induced proper subgraph $F$ of $G$. It is easy to see that every connected $r$-regular graph is $(r+1)$-col-critical.

Observation 2 For any col-vertex-critical graph $G$,

(i) $\operatorname{col}(G)=0$ if and only if $G \simeq K_{0}$,

(ii) $\operatorname{col}(G)=1$ if and only if $G \simeq K_{1}$,

(iii) $\operatorname{col}(G)=2$ if and only if $G \simeq K_{2}$, and

(iv) $\operatorname{col}(G)=3$ if and only if $G$ is a cycle.

Observation 3 For any graph $G$ and any element $x \in E(G) \cup V(G)$, if $\operatorname{col}(G-x)<\operatorname{col}(G)$ then $\operatorname{col}(G-x)=\operatorname{col}(G)-1$.

Observation 4 A graph $G$ is col-vertex-critical if and only if $\operatorname{col}(G-v)<\operatorname{col}(G)$ for every vertex $v$ in G.

Observation 5 Given any graph $G$, there is a col-critical subgraph $F$ of $G$ with $\operatorname{col}(G)=\operatorname{col}(F)=$ $\delta(F)+1$. In particular, if $G$ is col-critical then $\operatorname{col}(G)=\delta(G)+1$.

Proof: Recall that $\operatorname{col}(G)=\max \{\delta(H) \mid H \subseteq G\}+1$. Among the subgraphs $H$ of $G$ with $\operatorname{col}(G)=$ $\delta(H)+1$, let $F$ denote a minimal one, that is, $\delta\left(F^{\prime}\right)<\delta(F)$ for every proper subgraph $F^{\prime}$ of $F$. (This minimum exists since $G$ is finite.) Then $F$ is col-critical with $\operatorname{col}(F)=\delta(F)+1=\operatorname{col}(G)$. 
Observation 6 Given any graph $G$, there is a col-vertex-critical induced subgraph $F$ of $G$ with $\operatorname{col}(G)=$ $\operatorname{col}(F)=\delta(F)+1$. In particular, if $G$ is col-vertex-critical then $\operatorname{col}(G)=\delta(G)+1$.

Proof: Let $F$ denote a minimal induced subgraph of $G$ with $\operatorname{col}(F)=\operatorname{col}(G)$. This implies $\operatorname{col}\left(F^{\prime}\right)<$ $\operatorname{col}(F)$ for any induced proper subgraph $F^{\prime}$ of $F$, in particular, $F$ is a col-vertex-critical graph. Suppose $\operatorname{col}(F)>\delta(F)+1$. Then there is some proper induced subgraph $F^{\prime}$ of $F$ with $\delta\left(F^{\prime}\right)+1=\operatorname{col}(F)$, and so $\operatorname{col}\left(F^{\prime}\right) \geq \operatorname{col}(F)$, a contradiction. Hence $\operatorname{col}(F)=\delta(F)+1$. If $G$ is col-vertex-critical, then $F=G$, and the desired result follows.

The two following results may be of interest in their own right.

Proposition 1 (Pedersen [12]) For any two non-empty disjoint graphs $G_{1}$ and $G_{2}$, the colouring number of the complete join $G_{1}+G_{2}$ is at most

$$
\min \left\{\operatorname{col}\left(G_{1}\right)+n\left(G_{2}\right), \operatorname{col}\left(G_{2}\right)+n\left(G_{1}\right)\right\}
$$

and at least

$$
\min \left\{\operatorname{col}\left(G_{1}\right)+n\left(J_{2}\right), \operatorname{col}\left(G_{2}\right)+n\left(J_{1}\right)\right\}
$$

where, for each $i \in\{1,2\}, J_{i}$ is any subgraph of $G_{i}$ with minimum degree equal to $\operatorname{col}\left(G_{i}\right)-1$.

If, in addition, $\operatorname{col}\left(G_{i}\right)=\delta\left(G_{i}\right)+1$ for each $i \in\{1,2\}$ (in particular, if both $G_{1}$ and $G_{2}$ are colvertex-critical), then the colouring number of the complete join $G_{1}+G_{2}$ is equal to the minimum in (3).

A graph $G$ is said to be decomposable if there is a partition of $V(G)$ into two (non-empty) sets $V_{1}$ and $V_{2}$ such that, in $G$, every vertex of $V_{1}$ is adjacent to every vertex of $V_{2}$. Given any graph $G$, we let $V_{\delta}(G)$ denote the set of vertices of $G$ of minimum degree in $G$. Clearly, $V_{\delta}(G)$ is non-empty for any non-empty graph.

Proposition 2 (Pedersen [12]) Let $G$ denote a decomposable graph. Then $G$ is col-critical if and only if the vertex set of $G$ can be partitioned into two sets $V_{1}$ and $V_{2}$ such that $G=G_{1}+G_{2}$, where $G_{i}:=G\left[V_{i}\right]$ for $i \in\{1,2\}, G_{1}$ is regular, and

(i) $V\left(G_{2}\right) \backslash V_{\delta}\left(G_{2}\right)$ is an independent set of $G_{2}$, and

$$
\delta\left(G_{1}\right)+n\left(G_{2}\right)=\delta\left(G_{2}\right)+n\left(G_{1}\right)
$$

or

(ii) $G_{2}$ is an edgeless graph, and

$$
n\left(G_{1}\right)-\delta\left(G_{1}\right)-n(Q)<n\left(G_{2}\right)<n\left(G_{1}\right)-\delta\left(G_{1}\right)
$$

where $Q$ denotes a smallest component of $G_{1}$ (in terms of the number of vertices).

Moreover, $\operatorname{col}(G)=\delta\left(G_{1}\right)+n\left(G_{2}\right)+1$ in both (i) and (ii). 


\section{Double-col-critical graphs}

The analogue of the Double-Critical Graph Conjecture with $\chi$ replaced by col does not hold. For instance, the non-complete graph $C_{6}^{2}$ is 4-regular, 5-col-critical, and double-col-critical. Since $C_{6}^{2}$ is planar, it also follows that it is not even true that every double-col-critical graph with colouring number 5 contains a $K_{5}$ minor. (In [5], it was proved that every double-critical graph $G$ with $\chi(G) \leq 7$ at least contains a $K_{\chi(G)}$ minor.) It is easy to see that the square of any cycle of length at least 5 is a double-col-critical graph with colouring number 5 .

Observation 7 Any double-col-critical graph is col-vertex-critical.

Proof: Let $G$ denote a double-col-critical graph. If there are no vertices in $G$, then we are done. Let $v$ denote an arbitrary but fixed vertex of $G$. If there is no vertex in $G$ adjacent to $v$, then we are done, since then, by the connectedness of $G, G$ is just the singleton $K_{1}$. Let $u$ denote a neighbour of $v$. By Observation 4, we need to show $\operatorname{col}(G-v)<\operatorname{col}(G)$. The fact that $G$ is double-col-critical implies $\operatorname{col}(G-u-v) \leq \operatorname{col}(G)-2$. Suppose $\operatorname{col}(G-v) \geq \operatorname{col}(G)$. Then

$$
\operatorname{col}((G-v)-u) \leq \operatorname{col}(G)-2=\operatorname{col}(G-v)-2
$$

which contradictions Observation 3 This shows $\operatorname{col}(G-v)$ is strictly less than $\operatorname{col}(G)$, as desired.

Observation 8 For each integer $k \in\{0,1,2,3,4\}$, the only double-col-critical graph with colouring number $k$ is the $k$-clique.

Proof: Let $G$ denote a double-col-critical graph, and define $k:=\operatorname{col}(G)$. Then, by Observation $7, G$ is also col-vertex-critical, and so, by Observation $6, \delta(G)=k-1$. If $k \leq 3$, then the desired result follows immediately from Observation 2 Suppose $k=4$. Then, for any edge $e \in E(G), \operatorname{col}(G-V(e)) \leq 2$ and so, by Observation 1. $G-V(e)$ is a forest. Fix an edge $x y \in E(G)$. If $G-x-y$ contains no edges, then $G$ is 2 -degenerate and $\operatorname{so} \operatorname{col}(G) \leq 3$, a contradiction. Let $T$ denote a component of $G-x-y$ with at least one edge, and let $u$ and $v$ denote two leafs of $T$. Since, as noted above, $\delta(G)=3$, it follows that both $u$ and $v$ are adjacent to both $x$ and $y$. If $u$ and $v$ are adjacent in $T$, then $G[\{u, v, x, y\}] \simeq K_{4}$, and so, since $G$ is also col-vertex-critical, $G \simeq K_{4}$. Hence we may assume that $u$ has a neighbour $t$ in $T-v$. Now $G[\{x, y, v\}]$ is a 3 -clique in $G-t-u$ and $\operatorname{socol}(G-t-u) \geq \operatorname{col}(G[\{x, y, v\}])=3$, a contradiction. This completes the proof.

It is easy to verify that the graphs $Q_{1}, Q_{2}$, and $Q_{3}$ in Figure 1 are double-col-critical and have colouring number 5 . None of the graphs $Q_{1}, Q_{2}$, and $Q_{3}$ are squares of a cycle. We shall see that $Q_{1}, Q_{2}, Q_{3}$, and the squares of the cycles of length at least 5 are all the double-col-critical graphs with colouring number 5. First a few preliminary observations.

Observation 9 If $G$ is a double-col-critical graph, then $\delta(G)=\operatorname{col}(G)-1$ and every pair of adjacent vertices of $G$ has a common neighbour of degree $\delta(G)$ in $G$.

Proof: Let $G$ denote a double-col-critical graph. Then, by Observation $7, G$ is also col-vertex-critical, and so, by Observation $6, \delta(G)=\operatorname{col}(G)-1$. Let $x y$ denote an arbitrary edge of $G$. Now, by the definition of the colouring number, $G-x-y$ has minimum degree at most $(\operatorname{col}(G)-2)-1$ which is equal to $\delta(G)-2$. This means that some vertex of $V(G) \backslash\{x, y\}$, say $z$, which has degree at least $\delta(G)$ in $G$ has 


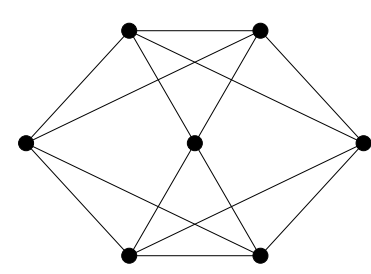

(a) The graph $Q_{1}$.

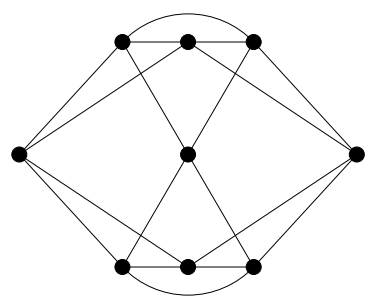

(b) The graph $Q_{2}$.

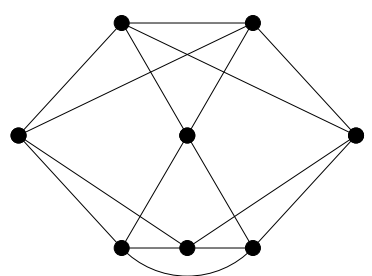

(c) The graph $Q_{3}$.

Fig. 1: The graphs $Q_{1}, Q_{2}$, and $Q_{3}$, depicted above, are the only double-col-critical graphs with colouring number 5 which are not squares of cycles.

degree at most $\delta(G)-2$ in $G-x-y$. The only way this can happen is if $z$ has degree $\delta(G)$ in $G$ and is adjacent to both $x$ and $y$ in $G$. This completes the argument.

Observation 10 If $G$ is a non-complete double-col-critical graph, then $G$ does not contain a clique of order $\operatorname{col}(G)-1$.

Proof: Let $G$ denote a non-complete double-col-critical graph. By Observation 7, $G$ is col-vertex-critical, and so, since $G$ is also non-complete, $G$ cannot contain a clique of order more than $\operatorname{col}(G)-1$. Also, by Observation 7, $\delta(G)=\operatorname{col}(G)-1$. Suppose that $G$ contains a clique $K$ of order $\operatorname{col}(G)-1$. Clearly, $G-V(K)$ is not empty. If $G-V(K)$ contains an edge $x y$, then $\operatorname{col}(G)-1=\operatorname{col}(K) \leq \operatorname{col}(G-x-y) \leq$ $\operatorname{col}(G)-2$, a contradiction. Hence $G-V(K)$ is edgeless, and so, since $\delta(G)=\operatorname{col}(G)-1=n(K)$, it follows that each vertex of $V(G) \backslash V(K)$ is adjacent to every vertex of $V(K)$, in particular, $G$ contains a clique of order $\operatorname{col}(G)$, a contradiction.

Proposition 3 Every double-col-critical graph with colouring number at least 3 is 2-connected.

Proof: Let $G$ denote a double-col-critical graph with $\operatorname{col}(G) \geq 3$. Since $G$ is col-vertex-critical, it is connected with $\operatorname{col}(G)=\delta(G)+1$. Suppose $G$ is not 2-connected, and let $x$ denote a cutvertex of $G$. Each component of $G-x$ has minimum degree at least $\delta(G)-1$. Let $C$ denote a component of $G-x$, and let $e$ denote some edge of $G-V(C)$. Then, using the fact that $G$ is double-col-critical, col is monotone, and $C$ is a subgraph of $G-V(e)$, we obtain

$$
\operatorname{col}(G)-1=(\delta(G)+1)-1 \leq \operatorname{col}(C) \leq \operatorname{col}(G-V(e))=\operatorname{col}(G)-2
$$

a contradiction. This shows that $G$ must be 2-connected.

\subsection{Double-col-critical graphs with colouring number 5 .}

We shall say that a $k$-neighbour of a vertex $x$ is a neighbour of $x$ of degree $k$.

Observation 11 If $G$ is a double-col-critical graph with colouring number 5 and $a b \in E(G)$, then a or $b$ has degree 4 in $G$. 
Proof: Let $G$ denote a double-col-critical graph with colouring number 5 . Then $\delta(G)=4$. Let $a b$ denote an edge of $G$. Suppose that both $a$ and $b$ have degree greater than 4 in $G$. By Observation 9 , there is a common 4-neighbour $c$ of $a$ and $b$. We shall make repeated use of Observation 9 and Observation 10 The latter observation implies that $G$ contains no 4-clique. There is a common 4-neighbour $d$ of $a$ and $c$. Since $\omega(G) \leq 3, d$ is not adjacent to $b$. This implies that there is a common 4-neighbour $e$ of $b$ and $c$ and $e$ is not identical to $d$. The vertex $e$ is not adjacent to $a$. The vertex $a$ has degree at least 5 , and so the common 4neighbour of $c$ and $d$ must be $e$. We note that $\{a, b, c, d, e\}$ induces a subgraph of $G$ of minimum degree 3 . Hence $G-\{a, b, c, d, e\}$ contains no edges. Moreover, $\operatorname{col}(G-\{c, d, e\}) \leq \operatorname{col}(G)-2$, and so $G-\{c, d, e\}$ contains a vertex $f$ of degree at most 2 . Since $a$ and $b$ both have degree at least 3 in $G-\{c, d, e\}$ and $N(c, G)=\{a, b, d, e\}$, it follows that this vertex $f$ must be in the set $V(G) \backslash\{a, b, c, d, e\}$ and that $f$ is adjacent both $d$ and $e$. The vertex $f$ has degree 4 in $G$. It also follows from the fact that $G-\{a, b, c, d, e\}$ contains no edges that $f$ must be adjacent to both $a$ and $b$. Since $a$ has degree at least 5 in $G$, it follows that $a$ must be adjacent to some vertex $g \in V(G) \backslash\{a, b, c, d, e, f\}$. Then $\operatorname{col}(G-a-g) \leq \operatorname{col}(G)-2=3$. On the other hand, $\{b, c, d, e, f\}$ induce a subgraph of $G-a-g$ of minimum degree 3 , a contradiction. This contradiction implies that $G$ contains no two adjacent vertices both of which have degree greater than 4. $\square$ Recently, the first author obtained a characterisation of what he called minimal critical graphs

with minimum degree 4 . It turns out that our double-col-critical graphs with colouring number 5 are such graphs, and so - using the characterisation of minimal critical graphs of minimum degree 4 - we obtain a characterisation of the double-col-critical graphs with colouring number 5 .

In the following result, which is the main result of this paper, we let $Q_{1}, Q_{2}$, and $Q_{3}$ denote the graphs depicted in Figure 1

Theorem 1 A graph is double-col-critical with colouring number 5 if and only if it is isomorphic to $Q_{1}$, $Q_{2}, Q_{3}$, or the square of a cycle of length at least 5 .

The graph $Q_{2}$ is the dual of the Herschel graph which is the smallest nonhamiltonian polyhedral graph.

In order to prove Theorem 1, we first need to introduce a bit of notation and state the above-mentioned characterisation of minimal critical graphs with minimum degree 4 .

For the remaining part of this section we shall be using the following notation. We shall let $\mathcal{C}$ denote the set of simple connected graphs of minimum degree at least 4 . An edge $e$ of a graph $G$ in $\mathcal{C}$ is essential if the graph $G-e$ obtained from $G$ by deleting $e$ is not in $\mathcal{C}$, and let us call $e$ critical if the graph $G / e$ obtained by contracting $e$ and simplifying is not in $\mathcal{C}$. An edge $e$ is essential if and only if $e$ is a bridge or at least one of its endvertices has degree 4; and $e$ is critical if and only if the endvertices of $e$ have a common 4-neighbour or $N(V(e), G)$ consists of three common neighbours of the endvertices of $e$. We are now interested in the minimal critical graphs in $\mathcal{C}$, that is, graphs $G \in \mathcal{C}$ with the property that each edge of $G$ is both essential and critical.

For the description of the minimal critical graphs in $\mathcal{C}$, we shall consider a number of bricks, that is, any graph isomorphic to one of the following nine graphs: $K_{5}, K_{2,2,2}, K_{5}^{-}, K_{2,2,2}^{-}, K_{5}^{\nabla}, K_{2,2,2}^{\nabla}, K_{5}^{\triangleright \triangleleft}$, $K_{2,2,2}^{\triangleright \triangleleft}$, or $K_{3}$ which are depicted in Figure 2 Each brick comes together with its vertices of attachment: For $K_{5}$ and $K_{2,2,2}$, this is an arbitrary single vertex, for the other seven bricks these are its vertices of degree less than 4 . The remaining vertices of the brick are its internal vertices, and the edges connecting two inner vertices are called its internal edges. Observe that every brick $B$ has one, two, or three vertices of attachment, and that they are pairwise nonadjacent unless $B$ is the triangle, that is, $K_{3}$.

It turns out that the minimal critical graphs from $\mathcal{C}$ are either squares of cycles of length at least 5 , or they are the edge disjoint union of bricks, following certain rules. This is made precise in the following 

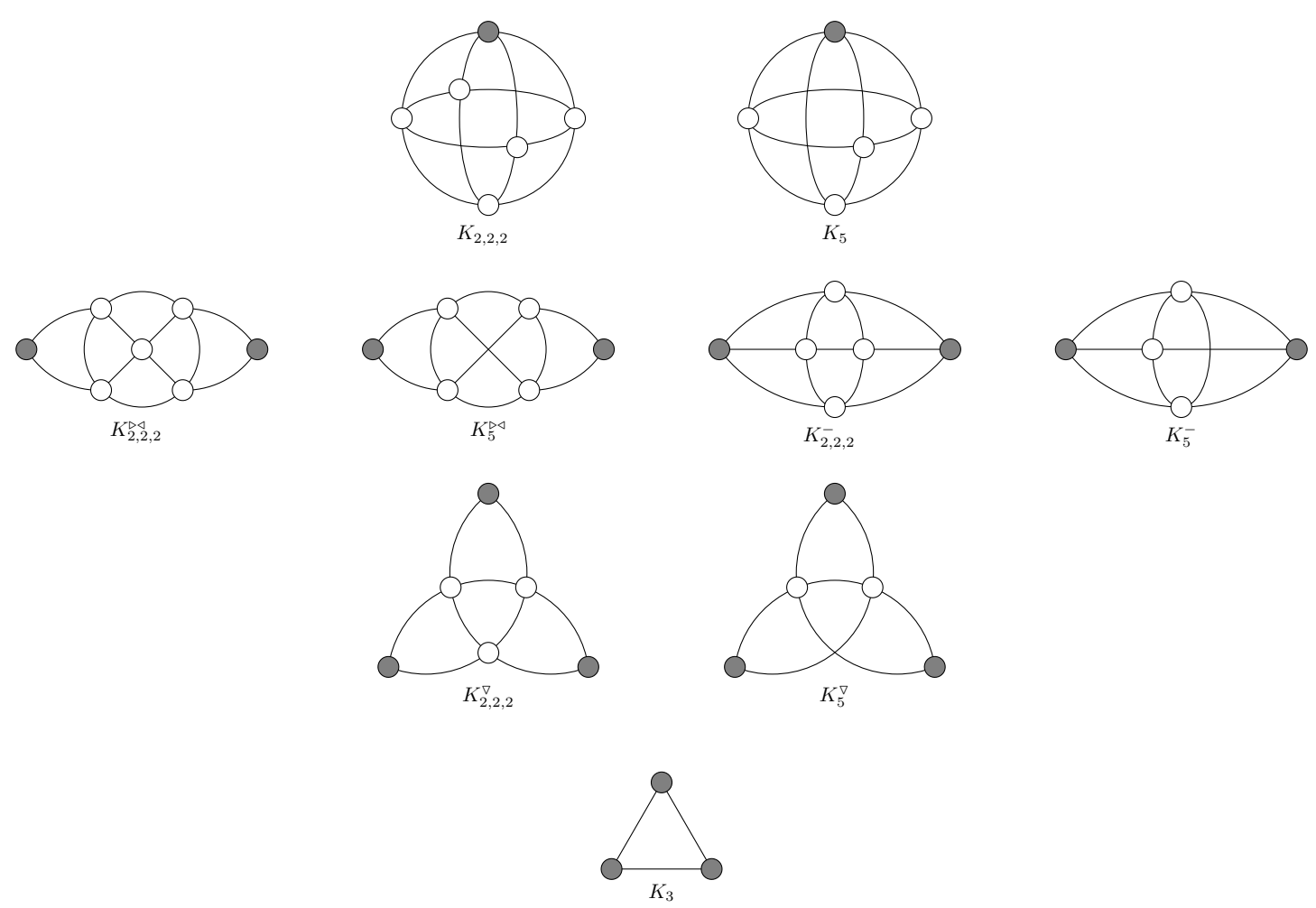

Fig. 2: The nine bricks. Vertices of attachment are displayed solid.

theorem.

Theorem 2 (Kriesell [7]) A graph is a minimal critical graph in $\mathcal{C}$ if and only if it is the square of a cycle of length at least 5 or arises from a connected multihypergraph $H$ of minimum degree at least 2 with at least one hyperedge and $|V(e)| \in\{1,2,3\}$ for all hyperedges e by replacing each hyperedge e by a brick $B_{e}$ (see Figure 2) such that the vertices of attachment of $B_{e}$ are those in $V(e)$ and at the same time the only objects of $B_{e}$ contained in more than one brick, and

(TB) the brick $B_{e}$ is triangular only if each vertex $x \in V(e)$ is incident with precisely one hyperedge $f_{x}$ different from e and the corresponding brick $B_{f_{x}}$ is neither $K_{5}, K_{5}^{-}, K_{2,2,2}$, nor $K_{2,2,2}^{-}$, and, for any other vertex $y \in V(e) \backslash\{x\}$ and hyperedge $f_{y}$ containing $y$ but distinct from $e$, we have

(i) $V\left(f_{x}\right) \cap V\left(f_{y}\right) \neq \emptyset$ only if not both of $B_{f_{x}}$ and $B_{f_{y}}$ are triangular, and

(ii) $f_{x}=f_{y}$ only if $B_{f_{x}}$ is $K_{5}^{\triangleright \triangleleft}$ or $K_{2,2,2}^{\triangleright \triangleleft}$.

Proof of Theorem 1: : In order to prove the desired result, we prove the following equivalent statement. 
A graph is double-col-critical with colouring number 5 if and only if it is the square of a cycle of length at least 5 or one of the three graphs obtained by taking the union of two graphs $G_{1}$ and $G_{2}$ such that $G_{i} \simeq K_{5}^{\nabla}$ or $G_{i} \simeq K_{2,2,2}^{\nabla}$ for $i \in\{1,2\}$ and $x \in V\left(G_{1}\right) \cap V\left(G_{2}\right)$ if and only if $x$ has degree 2 in $G_{1}$ and degree 2 in $G_{2}$.

The 'if'-part of the statement above is straightforward to verify and it is left to the reader.

Let $G$ denote an arbitrary double-col-critical graph with colouring number 5 . It follows from the definition of double-col-critical graphs, Observation 6, Observation 7, Observation 9, and Observation 11 that $G$ has the following properties.

(a) $G$ has minimum degree 4 ;

(b) if $x$ and $y$ are adjacent vertices, then at least one of them has degree 4;

(c) if $x$ and $y$ are adjacent vertices, then they have a common 4-neighbour; and

(d) if $x$ and $y$ are adjacent in $G$, then $G-x-y$ has no induced subgraph of minimum degree at least 3 .

By (a), $G$ is in $\mathcal{C}$. By (b), every edge of $G$ is essential, and, by (c), every edge of $G$ is critical. Thus, $G$ is minimal critical in $\mathcal{C}$, and so Theorem 2 applies. Suppose that $G$ is not the square of a cycle. Then $G$ has a representation by a multihypergraph $H$ as described in Theorem 2 .

If $e$ is a 1-hyperedge then the unique attachment vertex of the corresponding brick $B_{e}$ in $G$ is a cutvertex of $G$, a contradiction to Proposition 3 .

Suppose that there exists a 2-hyperedge $e$ with $V(e)=\{u, v\}$. If $B_{e}$ is $K_{5}^{\triangleright \triangleleft}$ or $K_{2,2,2}^{\triangleright \triangleleft}$ then $B_{e}-V(e)$ is an induced subgraph of $G$ of minimum degree 3 , and since the vertex $u \in V(e)$ has only two neighbours in that subgraph, there is an edge in $G-\left(V\left(B_{e}\right) \backslash V(e)\right)$, contradicting (d). If, otherwise, $B_{e}$ is $K_{5}^{-}$ or $K_{2,2,2}^{-}$then, by Theorem 2, $B_{e}$ is an induced subgraph of $G$ of minimum degree 3. By (a), there is a vertex in $V(G) \backslash V\left(B_{e}\right)$, and it has a neighbour in $V(G) \backslash V\left(B_{e}\right)$. This contradicts (d). Hence there are only 3 -hyperedges in $H$.

Suppose that $H$ contains a 3-hyperedge $e$ for which the corresponding brick $B_{e}$ is triangular. It follows from (a) and (d) that some vertex $q \in V(G) \backslash V\left(B_{e}\right)$ is adjacent to at least two vertices in $V\left(B_{e}\right)$. The vertex $q$ is not adjacent to all three vertices of $V\left(B_{e}\right)$, since otherwise $G\left[V\left(B_{e}\right) \cup\{q\}\right]$ would induce a 4-clique in $G$ which contradicts Observation 10 . Let $x$ and $y$ denote the neighbours of $q$ in $V\left(B_{e}\right)$. By Theorem 2 (TB), $x$ is incident to exactly one hyperedge $f_{x}$ different from $e$. Similarly, $y$ is incident to exactly one hyperedge $f_{y}$ different from $e$. If $f_{x}=f_{y}$, then, by Theorem 2 (TB.ii), $B_{f_{x}}$ is $K_{5}^{\triangleright \triangleleft}$ or $K_{2,2,2}^{\triangleright \triangleleft}$, in particular, $f_{x}$ is a 2-hyperedge, a contradiction. Hence $f_{x} \neq f_{y}$ and so, since $q \in V\left(f_{x}\right) \cap V\left(f_{y}\right)$, it follows from Theorem 2 (TB.i) that not both $B_{f_{x}}$ and $B_{f_{y}}$ are triangular bricks. The fact that $f_{x}$ and $f_{y}$ are distinct and $q \in V\left(f_{x}\right) \cap V\left(f_{y}\right)$ implies that $q$ is an attachment vertex of both $B_{f_{x}}$ and $B_{f_{y}}$. Since $q$ is adjacent to $x$ and both $q$ and $x$ are attachment vertices, it follows that $B_{f_{x}}$ must be triangular. Similarly, $B_{f_{y}}$ must be triangular, and so we have obtained a contradiction. This shows that each hyperedge in $H$ is of the type $K_{5}^{\nabla}$ or $K_{2,2,2}^{\nabla}$.

Let $e$ denote an arbitrary 3-hyperedge of $H$. If there are two vertices $x, y \in V(e)$ of degree exceeding 4 in $G$ then $G-\left(V\left(B_{e}\right) \backslash\{x, y\}\right)$ has minimum degree at least 3, contradicting (d) applied to any internal edge of $B_{e}$. Therefore, if there is a vertex $x \in V(e)$ of degree exceeding 4 in $G$, then the two vertices $y, z \in V(e) \backslash\{x\}$ are incident with precisely one further 3-hyperedge $f_{y}$ and $f_{z}$, respectively, both distinct 
from $e$. If $f_{y} \neq f_{z}$ then one may argue as above that $G-V\left(B_{e}-x\right)$ has minimum degree at least 3 , contradicting (d) applied to any internal edge of $B_{e}$. Hence $f_{y}=f_{z}=: f$. Let $w$ be the vertex in $V(f) \backslash\{y, z\}$. If $w \neq x$ then $\{w, x\}$ forms a 2-separator, and otherwise $w=x$ is a cutvertex as $x$ has degree exceeding 4 . In either case, $G-\left(V\left(B_{e}-x\right) \cup V\left(B_{f}\right)\right)$ has minimum degree at least 3 , again contradicting (d).

Hence all vertices of attachment have degree 4 in $G$. Let $e$ denote a 3-hyperedge in $H$, and let $x, y$, and $z$ denote the vertices of $V(e)$. Again let $f_{x}, f_{y}, f_{z}$ denote the unique 3-hyperedge distinct from $e$ incident with $x, y, z$, respectively. If they are pairwise distinct then $G-V\left(B_{e}\right)$ has minimum degree at least 3 , contradiction to (d). If $f:=f_{y}=f_{z} \neq f_{x}$ then let $w$ be the vertex in $V(f)$ distinct from $y, z$. As $f_{x} \neq f$, we have $w \neq x$, so that $G-(V(e) \cup V(f))$ has minimum degree at least 3, contradicting (d), unless there is a vertex in $V\left(f_{x}\right)$ adjacent to both $x$ and $w$; in this latter case, the unique 3-hyperedge distinct from $f$ incident with $w$ must be $f_{x}$, and so the vertex $u$ in $V\left(f_{x}\right) \backslash\{w, x\}$ is a cutvertex of $G$, a contradiction to Proposition 3 . Hence $f_{x}=f_{y}=f_{z}$, and the desired statement follows.

$\square$ Given our

success in characterising the double-col-critical graphs with colouring number 5 , we venture to ask for a characterisation of the double-col-critical graphs with colouring number 6 . If $G$ is a double-col-critical graph, then $G+K_{k}$ is a double-col-critical graph with $\operatorname{col}\left(G+K_{k}\right)=\operatorname{col}(G)+k$ (see Proposition 4p. This implies that the graphs $Q_{1}+K_{1}, Q_{2}+K_{1}, Q_{3}+K_{1}$, and $C+K_{1}$, where $C$ is the square of any cycle of length at least 5 , are all double-col-critical graphs with colouring number 6 . These are not the only double-col-critical graphs with colouring number 6 ; the icosahedral graph is yet another doublecol-critical graph with colouring number 6. This latter fact was also observed by Stiebitz [18, p. 323], although in a somewhat different setting. Using the computer programs SAGE [15] and geng [10], we determined all the double-col-critical graphs with colouring number 6 and at most 10 vertices; there are 116 such graphs and they are available at the second authors homepage [14].

The standard 6-regular toroidal graphs obtained from the toroidal grids by adding all diagonals in the same direction have colouring number 7 and are double-col-critical.

Complete joins of double-col-critical graphs. In [5], it was observed that if $G$ is the complete join $G_{1}+G_{2}$, then $G$ is double-critical if and only if both $G_{1}$ and $G_{2}$ are double-critical. Next we prove that the 'if'-part of the analogous statement for double-col-critical graphs is true. The 'only if'-part is not true, as follows from considering the double-col-critical graph $C_{6}^{2}$ : We have $C_{6}^{2} \simeq C_{4}+\overline{K_{2}}$ but neither $C_{4}$ nor $\overline{K_{2}}$ is double-col-critical.

Proposition 4 If $G_{1}$ and $G_{2}$ are two disjoint double-col-critical graphs, then the complete join $G_{1}+G_{2}$ is also double-col-critical with

$$
\operatorname{col}\left(G_{1}+G_{2}\right)=\min \left\{\operatorname{col}\left(G_{1}\right)+n\left(G_{2}\right), \operatorname{col}\left(G_{2}\right)+n\left(G_{1}\right)\right\}
$$

Proof: Let $G_{1}$ and $G_{2}$ denote two disjoint double-col-critical graphs. Then, by Observation 7 , both $G_{1}$ and $G_{2}$ are col-vertex-critical, and so, by Proposition 1 .

$$
\operatorname{col}\left(G_{1}+G_{2}\right)=\min \left\{\operatorname{col}\left(G_{1}\right)+n\left(G_{2}\right), \operatorname{col}\left(G_{2}\right)+n\left(G_{1}\right)\right\}
$$

We need to prove that $\operatorname{col}\left(\left(G_{1}+G_{2}\right)-x-y\right) \leq \operatorname{col}\left(G_{1}+G_{2}\right)-2$ for every edge $e=x y \in E(G)$; by symmetry, it suffices to consider (1) $x, y \in V\left(G_{1}\right)$ and (2) $x \in V\left(G_{1}\right)$ and $y \in V\left(G_{2}\right)$. Suppose 
$x, y \in V\left(G_{1}\right)$. Then

$$
\begin{aligned}
\operatorname{col}\left(\left(G_{1}+G_{2}\right)-x-y\right) & =\operatorname{col}\left(\left(G_{1}-x-y\right)+G_{2}\right) \\
& \leq \min \left\{\operatorname{col}\left(G_{1}-x-y\right)+n\left(G_{2}\right), \operatorname{col}\left(G_{2}\right)+n\left(G_{1}-x-y\right)\right\} \\
& \leq \min \left\{\operatorname{col}\left(G_{1}\right)-2+n\left(G_{2}\right), \operatorname{col}\left(G_{2}\right)+n\left(G_{1}\right)-2\right\} \\
& =\min \left\{\operatorname{col}\left(G_{1}\right)+n\left(G_{2}\right), \operatorname{col}\left(G_{2}\right)+n\left(G_{1}\right)\right\}-2
\end{aligned}
$$

where we applied Proposition 1 and the fact that $G_{1}$ is double-col-critical. A similar argument applies in case (2). We omit the details. $\square$ Proposition 4 and the fact that both $C_{6}^{2}$ and $K_{t}$ are double-col-critical

immediately implies the following result, which, in particular, shows that, for each integer $k \geq 6$, there is a non-regular double-col-critical graph with colouring number $k$.

Corollary 1 For any positive integer $t$, the graph $G_{t}:=C_{6}^{2}+K_{t}$ is a double-col-critical graph with $\operatorname{col}\left(G_{t}\right)=t+5, \delta\left(G_{t}\right)=n\left(G_{t}\right)-2$ and $\Delta\left(G_{t}\right)=n\left(G_{t}\right)-1$.

\section{Double-col-critical edges}

In [5], Kawarabayashi, the second author, and Toft initiated the study of the number of double-critical edges in graphs. In this section, we study the number of double-col-critical edges in graphs. Kawarabayashi, the second author, and Toft proved the following theorem for which we shall prove an analogue for the colouring number.

The complete join $C_{n}+K_{1}$ of a cycle $C_{n}$ and a single vertex is referred to as a wheel, and it is denoted $W_{n}$. If $n$ is odd, we refer to $W_{n}$ as an odd wheel.

Theorem 3 (Kawarabayashi, Pedersen \& Toft [5]) If $G$ denotes a 4-critical non-complete graph, then $G$ contains at most $m(G) / 2$ double-critical edges. Moreover, $G$ contains precisely $m(G) / 2$ doublecritical edges if and only if $G$ is an odd wheel of order at least 6.

The following result is just a slight reformulation of Theorem 3.

Corollary 2 If $G$ denotes a 4 -chromatic graph with no 4 -clique, then $G$ contains at most $m(G) / 2$ doublecritical edges. Moreover, $G$ contains precisely $m(G) / 2$ double-critical edges if and only if $G$ is an odd wheel of order at least 6 .

Proof: Let $G$ denote a 4-chromatic graph with no 4-clique. If $e=x y$ is a double-critical edge in $G$, then $e$ is a critical edge of $G$ and $x$ is a critical vertex of $G$. We remove non-critical elements from $G$ until we are left with a 4-critical subgraph $G^{\prime}$. At no point did we remove an endvertex of a double-critical edge. Thus, the number of double-critical edges in $G$ is equal to the number of double-critical edges in $G^{\prime}$. Clearly, $G^{\prime}$ is a non-complete graph, and so, by Theorem 3 , the number of double-critical edges in $G^{\prime}$ is at most $m\left(G^{\prime}\right) / 2$ which is at most $m(G) / 2$. The second part of the corollary now follows easily.

The following result - which is an analogue of Theorem 3 with the chromatic number replaced by the colouring number - extends Observation 8

Proposition 5 If $G$ denotes a 4-col-critical non-complete graph, then $G$ contains at most $m(G) / 2$ doublecol-critical edges. Moreover, $G$ contains precisely $m(G) / 2$ double-col-critical edges if and only if $G$ is a wheel of order at least 6 . 
Lemma 1 If $e$ and $f$ are two double-col-critical edges in a 4-col-critical non-complete graph, then e and $f$ are incident.

Proof: Let $G$ denote a col-critical graph with $\operatorname{col}(G)=4$. Then, by Observation 5 , $\delta(G)=3$. We must have $\omega(G) \leq 3$, since $G$ is a 4-col-critical non-complete graph.

Suppose $e$ is an arbitrary double-col-critical edge in $G$. Then $\operatorname{col}(G-V(e))=2$ which, by Observation 1 (iii), means that $G-V(e)$ is a forest containing at least one edge and, since $\delta(G)=3$, $\delta(G-V(e)) \geq 1$ and each leaf in $G-V(e)$ is adjacent to both endvertices of $e$ in $G$. Let $u$ and $v$ denote two leafs of $G-V(e)$. Now, if $G$ contains some double-col-critical edge $f$ which is not incident to $e$, then $G-V(f)$ contains no cycles, since $\operatorname{col}(G-V(f))=2$, and so $f$ is incident to both $u$ and $v$, which implies $G[\{u, v\} \cup V(e)] \simeq K_{4}$, a contradiction. This means that any two double-col-critical edges of $G$ are incident, and the proof is complete.

Proof of Proposition 5: Let $G$ denote a 4-col-critical non-complete graph. Then $n(G) \geq 5$ and, by Observation 5, $\delta(G)=3$ which implies $m(G) \geq\lceil n(G) \cdot \delta(G) / 2\rceil \geq 8$. By Lemma 1, we only have to consider two cases: (i) $G$ contains three incident double-col-critical edges $x y, y z$, and $x z$, or (ii) there is a vertex $v \in V(G)$ such that every double-col-critical edge of $G$ is incident to $v$. If (i) holds, then, since $m(G) \geq 8$, the desired statement follows. Suppose (ii) holds. Then the number of double-col-critical edges in $G$ is at most $\operatorname{deg}(v, G)$. We may assume that there is at least one double-col-critical edge, say, $v w$ in $G$. Suppose $G-v$ is disconnected. Then, since $\delta(G)=3$, each component of $G-v$ has minimum degree at least 2 , and so, in particular, some component of $G-v-w$ has minimum degree at least 2 . This, however, contradicts the fact that $G-v-w$ is a forest. Hence $G-v$ is connected. By Observation 3 , $\operatorname{col}(G-v) \geq 3$ and so, by Observation 1 (iii), $G-v$ contains a cycle. Hence $G-v$ is a connected graph with at least one cycle, and so $m(G-v) \geq n(G-v)$. Thus,

$$
m(G)=\operatorname{deg}(v, G)+m(G-v) \geq \operatorname{deg}(v, G)+(n(G)-1) \geq 2 \operatorname{deg}(v, G)
$$

which implies that the number of double-col-critical edges is at most $m(G) / 2$ and that the number of double-col-critical edges is equal to $m(G) / 2$ only if $\operatorname{deg}(v, G)=n(G)-1$ and $G-v$ is a cycle.

Conversely, if $G$ is a wheel on at least five vertices, then it is easy to see that exactly $m(G) / 2$ edges of $G$ are double-col-critical. This completes the proof. $\quad \square$ Let $k$ denote some integer greater

than 3 . Let $D_{k}$ denote the $2 k$-cycle with vertices labelled cyclically $v_{0} v_{1} \ldots v_{k} u_{k-1} u_{k-2} \ldots u_{1}$. Let $F_{k}$ denote the graph

$$
D_{k}^{2}-u_{1} v_{1}-u_{k-1} v_{k-1}+v_{1} v_{k-1}+u_{1} u_{k-1}
$$

Figure 3 depicts a drawing of $F_{5}$.

Observation 12 For every integer $k$ greater than 3 , the graph $F_{k}$, as defined as above, is a 5-col-critical graph with colouring number 5 in which all edges except $v_{1} v_{k-1}$ and $u_{1} u_{k-1}$ are double-col-critical.

Proposition 6 For each integer p greater than 4 and positive real number $\epsilon$, there is a p-col-critical graph $G$ with the ratio of double-col-critical edges between $1-\epsilon$ and 1 .

Proof: If $p=5$, the desired result follows directly from Observation 12 by letting $k$ tend to infinity. Let $p$ denote an integer greater than 5 and $\epsilon$ a positive real number. Let $k$ denote an integer a lot greater than $p$, and let $G$ denote the graph obtained by taking the complete join of $F_{k}$ and $\overline{K_{p-5}}$. Then, by 


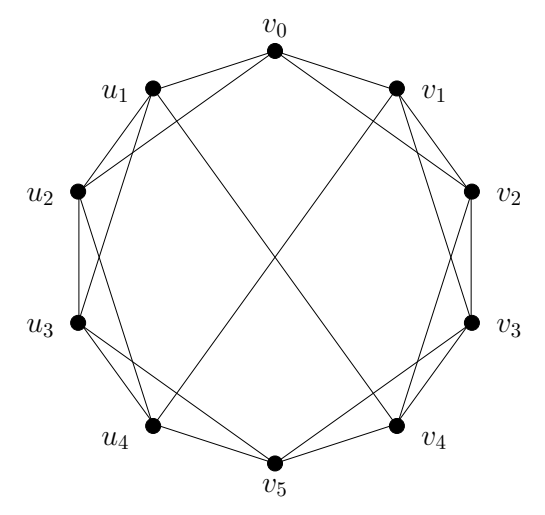

Fig. 3: The graph $F_{5}$ has colouring number 5 and all edges of $F_{5}$, except $u_{1} v_{4}$ and $v_{1} u_{4}$, are double-col-critical.

Proposition 2 (ii), $G$ is $p$-col-critical, and, since $k$ is a lot greater than $p$, all but the edges $v_{1} v_{k-1}$ and $u_{1} u_{k-1}$ are double-col-critical in $G$. By letting $k$ tend to infinity the ratio of double-col-critical edges in $G$ will from a certain point onwards be between $1-\epsilon$ and 1 . This completes the argument.

Proposition 6 means that there is no result corresponding to Proposition 5 for colouring numbers greater than 4 .

\section{Concluding remarks}

By a theorem of Mozhan [11] and, independently, Stiebitz [16], $K_{5}$ is the only double-critical graph with chromatic number 5 , that is, $K_{5}$ is the only 5 -chromatic graph with $100 \%$ double-critical edges, but we do not know whether there are non-complete 5 -chromatic graphs with the percentage of double-critical edges arbitrarily close to a 100. In [5], Kawarabayashi, the second author, and Toft conjectured that if $G$ is a 5 -critical non-complete graph, then $G$ contains at most $\left(2+\frac{1}{3 n(G)-5}\right) \frac{m(G)}{3}$ double-critical edges.

As we have seen, the story is a bit different for the colouring number. By Proposition 6 for $p=5$, there are non-complete graphs with colouring number 5 with the percentage of double-col-critical edges arbitrarily close to a 100 . This only makes Theorem 1 all the more interesting. By Theorem 1 , we are able to distinguish between graphs with colouring number 5 having $99.99 \%$ double-col-critical edges and those that have a $100 \%$ double-col-critical edges.

The problem of obtaining a concise structural description of the double-col-critical graphs with colouring number $k \geq 6$ remains open. Given any graph $G$, it can be decided in polynomial time whether or not $G$ is double-col-critical, since the colouring number itself can be computed in polynomial time. Nevertheless, given the structural complexity of the double-col-critical graphs with colouring number 6 mentioned on page 58, it seems likely that even the problem of obtaining a concise structural description of the double-col-critical graphs with colouring number 6 is non-trivial.

\section{Acknowledgements}

We thank Bjarne Toft for posing the problem of characterising the double-col-critical graphs and his many insightful comments on colouring and degeneracy of graphs. 


\section{References}

[1] J. A. Bondy and U. S. R. Murty. Graph Theory, volume 244 of Graduate Texts in Mathematics. Springer, New York, 2008.

[2] P. Erdôs. Problem 2. In Theory of Graphs (Proc. Colloq., Tihany, 1966), page 361. Academic Press, New York, 1968.

[3] P. Erdős and A. Hajnal. On chromatic number of graphs and set-systems. Acta Math. Acad. Sci. Hungar, 17: 61-99, 1966.

[4] T. R. Jensen and B. Toft. Graph Coloring Problems. Wiley-Interscience Series in Discrete Mathematics and Optimization. John Wiley \& Sons Inc., New York, 1995. A Wiley-Interscience Publication.

[5] K. Kawarabayashi, A. S. Pedersen, and B. Toft. Double-critical graphs and complete minors. Electron. J. Combin., 17(1):Research Paper 87, 27 pp., 2010.

[6] M. Kriesell. A survey on contractible edges in graphs of a prescribed vertex connectivity. Graphs Combin., 18 (1):1-30, 2002.

[7] M. Kriesell. Nonseparating $K_{4}$-subdivisions in graphs of minimum degree at least 4 . Technical Report arXiv:1101.5278, Jan 2011. IMADA-preprint, 25 pages.

[8] D. R. Lick and A. T. White. k-degenerate graphs. Canad. J. Math., 22:1082-1096, 1970.

[9] D. W. Matula and L. L. Beck. Smallest-last ordering and clustering and graph coloring algorithms. J. Assoc. Comput. Mach., 30(3):417-427, 1983.

[10] B. D. McKay. geng - a program for generating graphs, 2008. URL http://cs.anu.edu . au/ bdm/ nauty/. Version 2.4.

[11] N. N. Mozhan. Twice critical graphs with chromatic number five. Metody Diskret. Analiz., (46):50-59, 73, 1987.

[12] A. S. Pedersen. Contributions to the Theory of Colourings, Graph Minors, and Independent Sets. PhD thesis, University of Southern Denmark, 2010.

[13] A. S. Pedersen, September 2012. URL http://www.imada.sdu.dk/ asp/ noSmallNonCompleteDC.pdf

[14] A. S. Pedersen, October 2012. URL http://www.imada.sdu.dk/ asp/double-col-critical. php.

[15] W. A. Stein et al. Sage Mathematics Software (Version 4.6.2). The Sage Development Team, 2012. http://www.sagemath.org.

[16] M. Stiebitz. $K_{5}$ is the only double-critical 5-chromatic graph. Discrete Math., 64(1):91-93, 1987.

[17] M. Stiebitz. On $k$-critical $n$-chromatic graphs. In Combinatorics (Eger, 1987), volume 52 of Colloq. Math. Soc. János Bolyai, pages 509-514. North-Holland, Amsterdam, 1988.

[18] M. Stiebitz. Decomposing graphs under degree constraints. J. Graph Theory, 23(3):321-324, 1996.

[19] B. Toft. Colouring, stable sets and perfect graphs. In Handbook of Combinatorics, Vol. 1, 2, pages $233-288$. Elsevier, Amsterdam, 1995. 